\title{
Spectral Identification of Specific Photophysics of Cy5 by Means of Ensemble and Single-Molecule
} Measurements

\author{
Zhengxi Huang Dongmei Ji Sufang Wang Andong Xia*
}

State Key Laboratory of Molecular Reaction Dynamics, Institute of Chemistry, Chinese Academy of Sciences, Beijing, 100080, P. R. China

Felix Koberling* Matthias Patting Rainer Erdmann

PicoQuant GmbH, Rudower Chausse 29, 12489 Berlin, Germany

andong@iccas.ac.cn; Koberling@pq.fta-berlin.de

\section{Supporting Information}

Figure S1 shows the typical on- and off-time histograms extracted from a single "on-state" molecule. Table S1 lists the detected count rate $\mathrm{I}_{\mathrm{det}}$, the on-state average counts $\mathrm{N}_{\text {on }}$ and triplet-state transition yield $\mathrm{Y}_{\text {isc }}$ of a single Cy5 molecule in the on-state and dim-state under continuous excitation at 632.8 nm. All parameters are calculated similar to refs.[1-3]. When a molecule enters a dim-state, there are no obvious changes in the average on- and off-times compared to that of the on-state, but with a different excitation rate and emission yield. Furthermore, this molecule displays an almost constant fluorescence lifetime around $2.0 \mathrm{~ns}$ independence of being in an on- or dim-state. This may indicate that both onstate and dim-state exhibit similar intersystem crossing and triplet properties. The longer fluorescence lifetime of a single Cy5 molecule with about $2.0 \mathrm{~ns}$, might be explained by a reduced flexibility of the 
molecules on the glass surface in comparison to the one measured from the Cy5 molecules in solution. Meanwhile, From the experimental results, we find that the two fluorescence decays from both solved Cy5 and surface-adsorbed Cy5 can be described by mono-exponential fit with decay time about $1.0 \mathrm{~ns}$ for solved Cy5 and about $2.0 \mathrm{~ns}$ for Cy5 both on the glass surface and in polymer matrix. It is found that single Cy5 molecules adsorbed on glass surfaces show stationary transition dipoles, leading to narrow distribution of fluorescence lifetime around $2 \mathrm{~ns}$, which are also in accordant with those results reported by refs.[3, 4]. If there is any electron transfer between Cy5 and glass surface, the fluorescence lifetime of Cy5 on the dry glass surface should be much shorter than that in solution. ${ }^{5}$ Therefore, the electron transfer between $\mathrm{Cy} 5$ and glass surface should play a limited and minor role in the observed behaviors. Figure S2 shows the ensemble-averaged absorption spectra of both solved and surface absorbed Cy5 molecules. It is found that the ensemble-averaged absorption spectrum of Cy5 on glass surface shows similar spectral shape to that of Cy5 in solution with a slight redshift, which results from aggregation (We also find that the absorption peak of very dilute Cy5 molecule on surface is just the same as that in solution. Unfortunately, we cannot get a good spectral shape of absorption of Cy5 on surface because of the limited sensitivity of UV-Vis spectrometer). Since the spectra of both solved and surface absorbed Cy5 molecules are similar, the spectral data of Cy5 obtained in solution from laser photolysis could still be suitable to be used for interpreting the underlying mechanisms of those unexpected single molecule behaviors. 

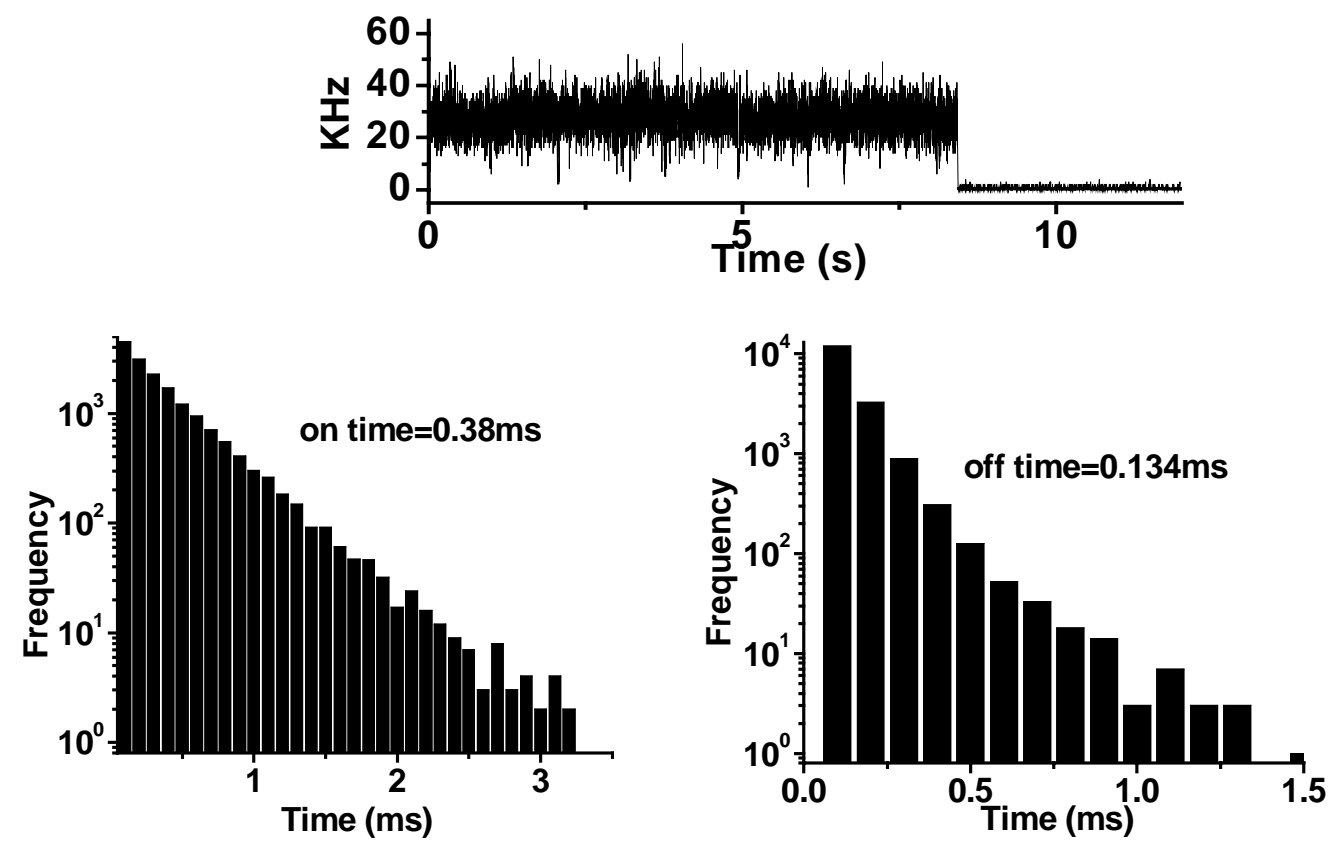

Figure S1. Typical fluorescence fluctuation with $1 \mathrm{~ms} / \mathrm{bin}$, and on-time and off-time histograms of a single "on-state" molecule. For the on-time and off-time data analysis, a bin width of $100 \mu$ s was used.

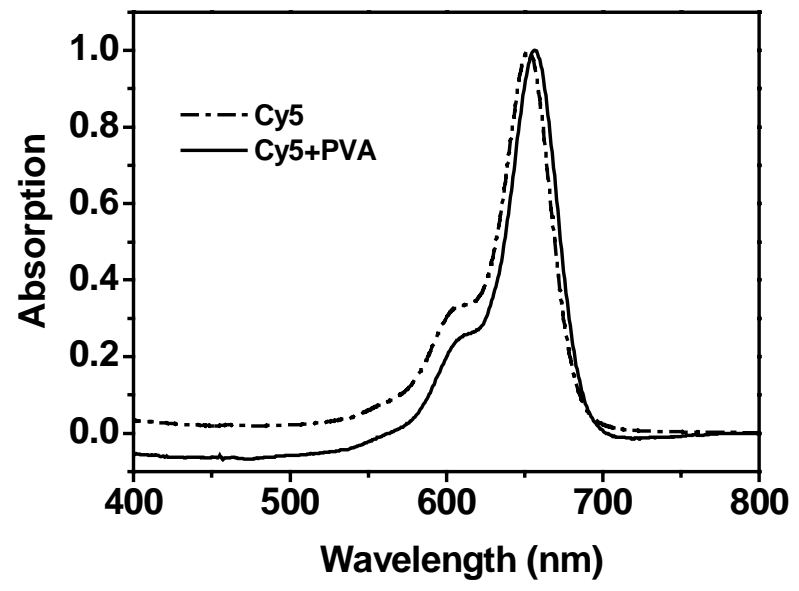


Figure S2 The ensemble-averaged absorption spectra of both solved and surface absorbed Cy5 molecules.

For those molecules with a long on time as shown in Figure 2(a) in text, the intensity time course under different excitation intensities from the same one individual molecule could be observed. The shortened off-times, increased on-times and increased total average photon counts are an obvious indication of photoinduced reverse intersystem crossing. The basic processes for this point can be explained as follows. Imagine an organic fluorophore with normal five-level model (see Figure S3), which is excited by an initial pulse from the ground to the first singlet state $S_{0} \rightarrow S_{1}$, the resulting $S_{1}$ state would usually decay to the ground state whereby a fluorescence photon is emitted, or it undergoes intersystem crossing to its triplet state $S_{1} \rightarrow T_{1}$, the resulting $T_{1}$ will go back to the ground state or transit to higher triplet state $\mathrm{T}_{\mathrm{n}}$ upon suitable excitation. Let's now assume that the molecule is also exposed to a second pulse or to a $\mathrm{CW}$ laser source with a wavelength in resonance with the $\mathrm{T}_{1} \rightarrow \mathrm{T}_{\mathrm{n}}$ transition. Once the molecule enters this $T_{1}$ state, the photoinduced reverse intersystem crossing by the sequence $\mathrm{T}_{1} \rightarrow \mathrm{T}_{\mathrm{n}} \rightarrow \mathrm{S}_{1} \rightarrow \mathrm{S}_{0}$ will be quite efficient as indicated by two-color double resonance experiments. $^{6-8}$ In our case, the excitation wavelength used for single molecule fluorescence measurement is just well in resonance with the $T_{1} \rightarrow T_{n}$ absorption as evidenced by laser flash photolysis mentioned in Text. The lifetime of $T_{n}$ is about 200fs, and the time of the transition from $T_{n}$ via $S_{n}(n \geq 1)$ to $S_{0}$ is less than $10 \mathrm{~ns}^{6-10}$ As a result, the triplet state can be excited $\left(T_{1} \rightarrow T_{n}\right)$ efficiently upon CW laser excitation at higher intensity, leading via RISC to a shortening in the off-time, an increasing in the total average photon counts and an increasing in the on-times. Furthermore, we also reduced the repetition of the laser excitation from $\mathrm{CW}$ laser to pulsed laser at a stronger average power below saturation. The laser excitation with low repetition is expected to reduce the $T_{1} \rightarrow T_{n}$ excitation efficiency, a decreasing in the on-time and total average photon counts, as well as an increasing in the off-time were observed obviously when the repetition of excited laser is reduced (data not shown). 
Table S1 The detected count rate $\left(\mathrm{I}_{\mathrm{det}}\right)$, average on-time and dim-time, on-state average counts $\left(\mathrm{N}_{\mathrm{on}}\right)$, off-state yield $\left(\Phi_{\text {off }}\right)$ and triplet-state transition yield $\left(\mathrm{Y}_{\text {isc }}\right)$ of single Cy5 molecules excited with CW. The value labeled with asterisk indicates that the value is smaller than the bin time; it is expected to have large error.

\begin{tabular}{|c|c|c|}
\hline & on state & dim state \\
\hline $\mathrm{I}_{\mathrm{det}}(\mathrm{count} / \mathrm{ms})$ & $21-48$ & $5-27$ \\
\hline on time $(\mathrm{ms})$ & $0.11-1.36$ & $0.03 *-0.37$ \\
\hline off time $(\mathrm{ms})$ & $0.074^{*}-0.53$ & $2-13$ \\
\hline $\mathrm{N}_{\text {on }}$ & $6-31$ & $(0.1-1.5) \times 10^{-2}$ \\
\hline$\Phi_{\text {off }}$ & $(0.2-1.0) \times 10^{-3}$ & $(0.39-2.5) \times 10^{-2}$ \\
\hline $\mathrm{Y}_{\text {isc }}$ & $(1.6-8.3) \times 10^{-3}$ & \\
\hline
\end{tabular}

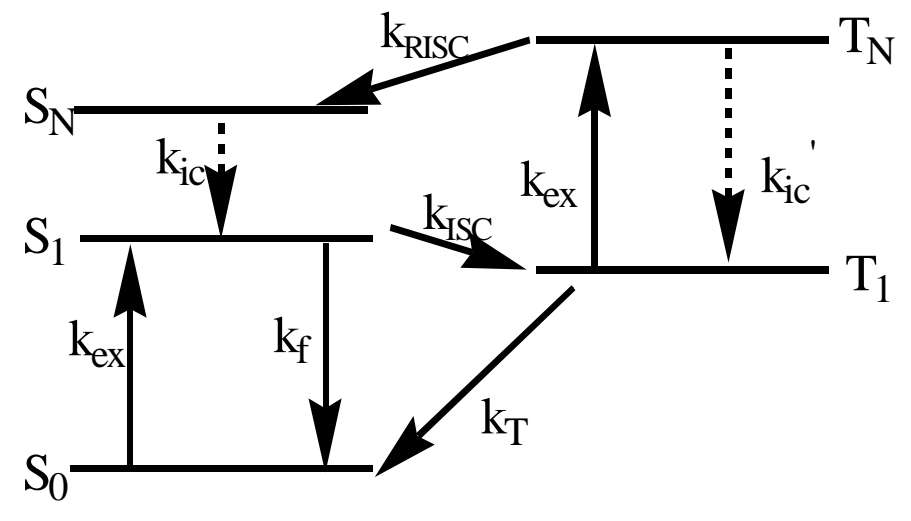


Figure S3. Scheme of the relevant five-level model and transitions for single trans-Cy5 molecule excited under high intensity.

Figure S4 shows the weighted residuals of the fitting for the data obtained by laser photolysis. The reduced Chi2 value for each fitting is also listed.

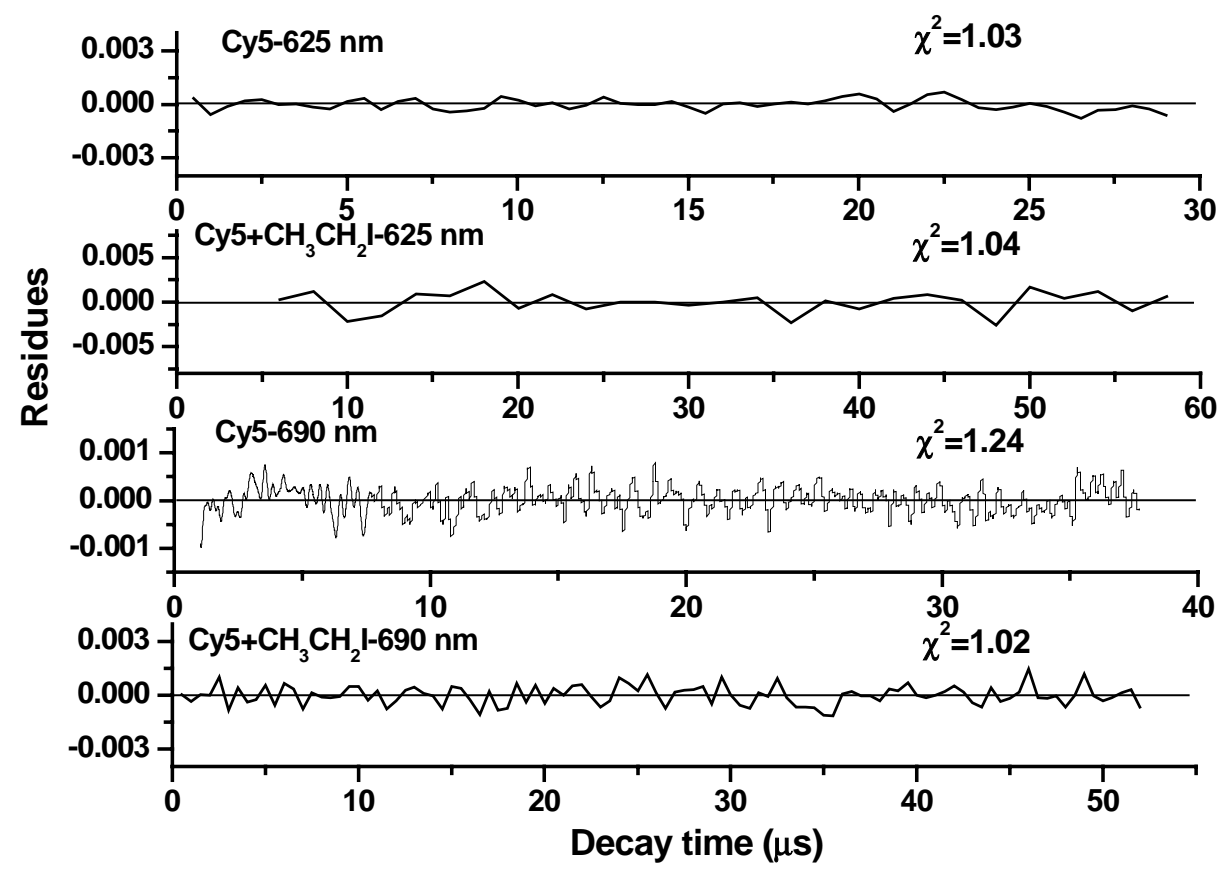

Figure S4 The weighted residuals of the fitting for the data obtained by laser photolysis. The reduced Chi2 value for each fitting is also listed.

Figure S5 shows the fluorescence spectrum of donor molecule TMR and absorption spectrum of acceptor molecule Cy5. It is found that the main overlapping region between the fluorescence spectrum of TMR and the absorption spectrum of Cy5 locates right in the region of absorption of trans-Cy5. 
There is no fluorescence of TMR above 660nm, where the absorption of cis-Cy5 locates. Therefore, it is obvious that cis-Cy5 cannot absorb the energy transferred from donor TMR.

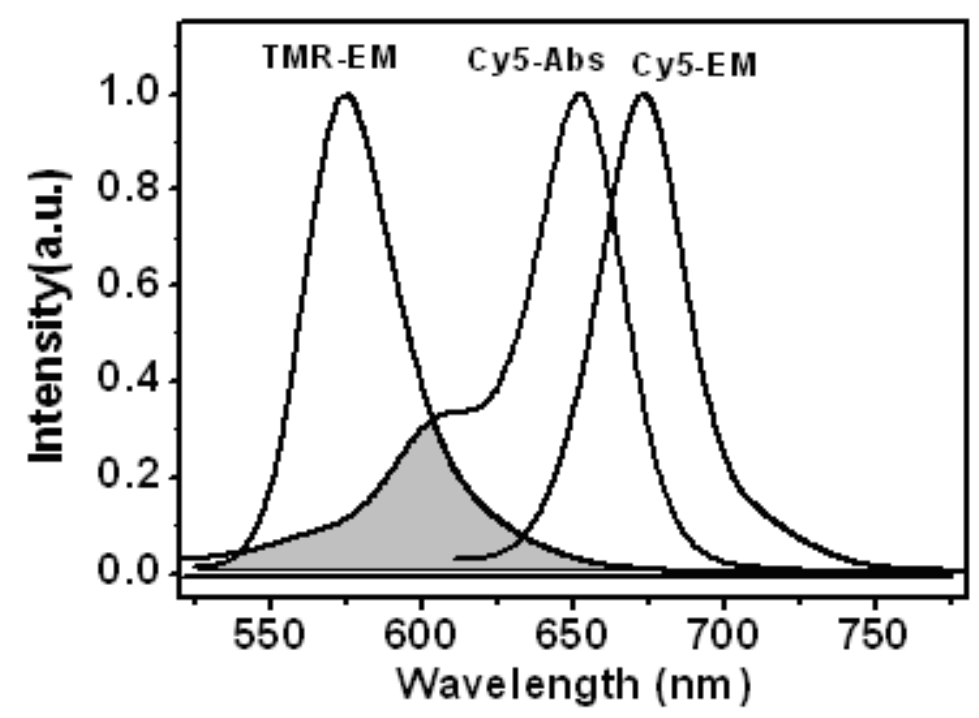

Figure S5. Normalized fluorescence spectrum of donor molecule TMR and absorption spectrum of acceptor molecule Cy5. The shaded area show the spectral overlap.

Finally, it should be mentioned that we performed all the experiments for Cy5 in $\mathrm{N}_{2}$-saturated atmosphere in order to ensure both a better observation of triplet dynamics and a large increase in the photochemical stability. In fact, the oxygen quenching for the triplet state of $\mathrm{Cy} 5$ is believed to produce singlet oxygen which was monitored from singlet oxygen phosphorescence at $1270 \mathrm{~nm}$ after excitation of an aerated solution at $632.8 \mathrm{~nm}$ as shown in Figure S6. The singlet oxygen spectrum was measured with a MagNA-IR 760 Nicolet Spectrometer equipped with a LN-Ge Detector. Taking TPP as a reference with $\varphi_{\text {TPP }}=0.62,{ }^{11}$ the singlet oxygen quantum yield of Cy5 $\left(\varphi_{\mathrm{Cy} 5}\right)$ was estimated to be about 0.084. The indeed singlet oxygen formation of Cy5 during excitation might have an influence on biological applications when Cy5 was used as fluorescence probe. 


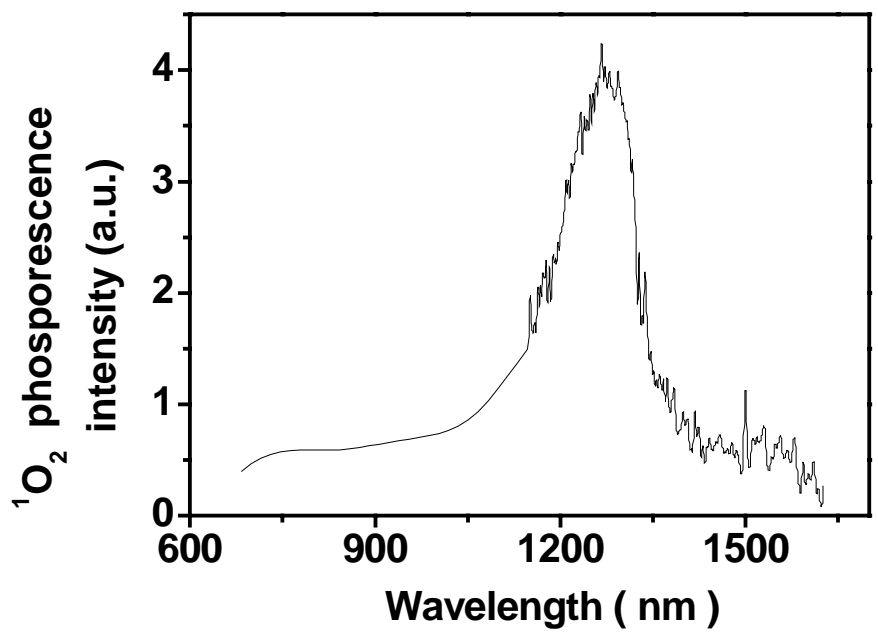

Figure S6. Singlet oxygen phosphorescence from an air-saturated Cy5-ethnaol solution $\left(0.5 \times 10^{-5} \mathrm{M}\right)$ excited at $632.8 \mathrm{~nm}$.

Acknowledgment We sincerely thank Prof. Desheng Jiang for assistance with singlet oxygen luminescence measurements.

\section{References}

[1]Veerman, J. A.; Garcia-Parajo, M. F.; Kuipers, L. and Van Hulst, N. F. Phys Rev Lett. 1999, 83, 2155-2158.

[2] Ha, T.; Enderle, Th.; Chemla, D. S.; Selvin, P. R.; Weiss, S. Chem. Phys. Lett. 1997, 271, 1-5.

[3] Tinnefeld, P.; Herten, D. P.; Sauer, M. J. Phys. Chem. A 2001, 105, 7989-8003.

[4] Tinnefeld, P.; Buschmann, V.; Herten, D. P.; Han, K. T.; Sauer, M. Single Mol. 2000, 1, $215-$ 223.

[5] Lu, H. P.; Xie, X. S. J. Phys. Chem. B 1997, 101, 2753-2757.

[6] English, D. S.; Harbron, E. J.; Barbara, P. F. J. Phys. Chem. A 2000, 104, 9057-9061. 
[7] Redmond, R. W.; Kochevar, I. E.; Krieg, M.; Smith, G.; McGimpsey, W. G. J. Phys. Chem. A 1997, 101, 2773-2777.

[8] Reindl, S.; Penzkofer, A. Chem. Phys. 1996, 21, 431-439.

[9] Eggeling, C.; Widengren, J.; Rigler, R.; Seidel, C. A. M. Anal. Chem. 1998, 70, 2651-2659.

[10] Larkin, J. M.; Donaldson, W. R.; Foster, T. H.; Knox, R. S. Chem. Phys. 1999, 244, 319-330.

[11] Mclean, A. J.; Mc Garvey, D. J.; Truscott, T. G.; Lambert, C. R. and Land, E. J. J. Chem. Soc. Faraday Trans. II 1990, 86, 3075-3078. 